Research paper

\title{
Daytime and outcomes after cardiac surgery: Systematic review and metaanalysis, insights from a large UK database review and post-hoc trial analysis
}

\author{
Daniel Paul Fudulu*, Arnaldo Dimagli, Lauren Dixon, Manraj Sandhu, Lucia Cocomello, \\ Gianni D Angelini, Umberto Benedetto
}

Bristol Heart Institute, University of Bristol, Upper Maudlin St, Bristol BS2 8HW UK

\section{A R T I C L E I N F O}

Article History:

Received 6 March 2021

Revised 9 April 2021

Accepted 28 April 2021

Available online 7 June 2021

\begin{abstract}
A B S T R A C T
Background: Several studies have suggested a variation of myocardial tolerance to ischaemia depending on the daytime of surgery. To test this hypothesis, we conducted a three-level analysis: metaanalysis, national patient-level dataset analysis and a post-hoc trial analysis.

Methods: We first performed a systematic review and metaanalysis of available studies comparing clinical outcomes following cardiac surgery performed in the morning (am) versus afternoon (pm). Then, we interrogated the UK national adult cardiac surgery audit database (NACSA) and analysed the am or pm outcomes of patients undergoing non-emergency aortic valve replacement (AVR) or coronary artery bypass grafting (CABG). In a post-hoc analysis, we further investigated the effect of time of surgery on serum troponin release and ventricular myocardial biopsy adenine nucleotide metabolism.

Results: A total of 18377 patients undergoing uncomplicated isolated CABG or isolated AVR on the same day am or pm were included in the metaanalysis. Meta-analytic estimates showed no difference in the risk of MI between patients operated in pm vs am (OR 1.02, 95\% CI:0.79-1.32) and in the risk of mortality (OR 1.1, 95\% $\mathrm{CI}: 0.85-1.42$ ). Outcomes of 91248 patients from the NACSA dataset were analysed according to the daytime of the procedure. Patient-level analysis showed no significant effect of daytime for both isolated AVR $(p=0.094)$ and isolated CABG ( $p=0.425)$. Finally, we performed a post-hoc trial database analysis in 124 patients undergoing isolated AVR or CABG of serial cardiac troponin and nucleotides metabolism on ventricular myocardial biopsies. We found no significant diurnal changes in the perioperative cardiac troponin release or nucleotide metabolism in the AVR $(p=0.30)$ or the CABG cohort $(p=0.97)$.

Conclusion: The present three-level analysis found no evidence that daytime influences clinical outcomes and myocardial injury in patients undergoing cardiac surgery.

(c) 2021 The Author(s). Published by Elsevier Ltd. This is an open access article under the CC BY-NC-ND license
\end{abstract}

(http://creativecommons.org/licenses/by-nc-nd/4.0/)

\section{Introduction}

Circadian rhythms influence the function of all body systems, including the cardiovascular system. One well-known example is the circadian variation in the frequency of onset of acute myocardial infarctions (MI) that occur more often in the morning (am) as compared to afternoon (pm) [1]. Mouse models of acute myocardial infarction demonstrated that there is a potential biological rhythm that regulates a higher ischaemic-reperfusion tolerance in the afternoon that translates into smaller infarct sizes. This rhythm appears to be governed by peripheral, cardiomyocyte clock genes since the

\footnotetext{
DOI of original article: http://dx.doi.org/10.1016/j.lanepe.2021.100143.

* Corresponding author.

E-mail address: daniel.fudulu@bristol.ac.uk (D.P. Fudulu).
}

genetic ablation of the cardiomyocyte clock genes abolishes this variation [2].

Cardiac surgery offers a predictable myocardial injury, hence a valuable research model. One landmark study suggested possible myocardial protection of cardiac surgery performed in the pm and that myocardial injury could be transcriptionally regulated by circadian clock genes [3]. Other mechanisms involved include the circadian variation of antioxidant hormones, such as melatonin [4]. This question is relevant because the time of surgery can be a useful parameter to improve risk prediction after heart surgery. Following Montaigne et al. study [3], several retrospective studies have reported contradicting results in terms of any effect of daytime of surgery on clinical outcomes [5-8]. To further investigate the above hypotheses, we have conducted a three-level analysis: we have first performed a systematic review and metaanalysis of all the available 


\section{Research in context}

\section{Evidence before this study}

We have performed a systematic review and metanalysis systematic review and metaanalysis of available studies comparing clinical outcomes following cardiac surgery performed in the morning (am) versus afternoon (pm). The search was conducted on 25 October 2020 in Ovid Embase, Ovid Medline, PubMed, Web of Science and Cochrane library. A Boolean logic search was used as follows: "((daytime) OR (diurnal) OR (circadian) OR (afternoon) OR (morning)) AND ((cardiac surgery) OR (heart surgery)) AND ((outcomes) OR (myocardial protection) OR (cardioprotection) OR (myocardial injury) OR (troponin))". A total of 965 references were identified through electronic database searches and reference lists. We included a total of five studies in the pooled analysis. One substudy was a randomized controlled trial, and the rest of the studies were propensity-matched. The risk o bias was low for all studies. All studies were from outside the UK. Meta-analytic estimates showed no difference in the risk of MI between patients operated in pm vs am (OR 1.02, 95\% CI:0.79-1.32) and in the risk of mortality (OR 1.1, 95\% Cl:0.85-1.42).

\section{Added value of this study}

We conducted an analysis of a large dataset from the UK. This patient-level study showed no significant effect of daytime for both isolated AVR and isolated CABG. This is in line with the available evidence synthesized in our metaanalysis. Also, we performed a post-hoc trial database analysis in patients undergoing isolated AVR or CABG of serial cardiac troponin and nucleotides metabolism on ventricular myocardial biopsies. We found no significant diurnal changes in the perioperative cardiac troponin release in blood or nucleotide metabolism in the cardiac tissue.

\section{Implications of all the available evidence}

Daytime of surgery does not seem to affect mortality, myocardial infarction rates or myocardial tolerance to ischaemia after cardiac surgery.

studies reporting on outcomes after cardiac surgery in am and pm. Secondly, we have performed a patient-level analysis of a national dataset, the UK National Adult Cardiac Surgery Audit (NACSA). Finally, we conducted a post-hoc trial analysis of the remote ischaemic preconditioning (RIPC) trial to assess the impact of daytime of the procedure on myocardial injury as measured by serial cardiac troponin and myocardial energy metabolites and phosphorylation potential in ventricular myocardial biopsies [9].

\section{Methods}

\subsection{Systematic review and metaanalysis}

\subsection{Eligibility criteria}

Studies included in the present metaanalysis met: (i) am and pm cohort comparison of patients undergoing heart surgery, out of working hours cohorts excluded (ii) study design: propensity-matched and randomised control trials. Non-English language review articles and editorials were excluded.

\subsection{Search strategy}

A Boolean logic search (refer to Supplementary material) was conducted on 25 October 2020 in Ovid Embase, Ovid Medline, PubMed, Web of Science and Cochrane library. References from the selected studies were also manually searched. Two reviewers (D.F. and L.D.) independently screened all studies for inclusion using the Rayyan platform (Ouzzani et al. 2016). Discrepancies were resolved by consensus. For articles selected for inclusion in the study, reference lists were also screened. A total of 965 references were identified through electronic database searches and reference lists. We included a total of five studies included in the analysis (Supplementary Figure 1). The quality of the included studies was assessed with the Newcastle-Ottawa scale for observational studies and with the Cochrane risk-of-bias tool for RCTs (RoB 2) by two reviewers (L.D and M.S.) (Supplementary Table 1).

\subsection{Data extraction}

Two reviewers (D.F. and L.C.) extracted data independently. Conflicts in extracted data were resolved by consensus. The extracted items included patient baseline characteristics, operative data, and postoperative outcomes. The characteristics of the studies included are summarised in Supplementary Tables 2 and 3.

\subsection{Outcomes}

Outcomes of interest were 30-day mortality and MI. Definitions of mortality and myocardial infarction used by each study are reported as Supplementary Material (Supplementary Table 4). We limited our metaanalysis to these two endpoints as other outcomes were inconsistently reported amongst studies, and they were not relevant to our hypothesis testing.

\subsection{Statistical analysis}

Measurement data were reported as the number of events for binary outcomes. We have conducted our metanalysis solely on adjusted outcomes of the propensity or randomised data. A random-effect model was used to compute the pooled odds ratio (OR) with a 95\% confidence interval [10]. Heterogeneity across studies that could not be attributable to chance alone was quantified, calculating the $\mathrm{I}^{2}$ value. We considered significant heterogeneity if $\mathrm{I}^{2}>75 \%$. Funnel plots were generated to evaluate publication bias (i.e., funnel asymmetry) with Egger's test. In the absence of heterogeneity, we have used a fixed-effect model. For studies with a zero-cell count, a standard continuity correction was applied in order to perform the inverse variance pooling [11]. All analyses were performed in $\mathrm{R}$ version 4.0.0. by using meta and metaphor packages (https://www.r-project.org/).

\subsection{NACSA audit dataset analysis}

The study was approved by the Health Research Authority (HRA) and Health and Care Research Wales (HCRW), and a waiver for patients' consent was obtained (IRAS ID: 278171). The NACSA registry prospectively collects demographic, as well as pre-, peri- and postoperative clinical information, including mortality, for all significant adult cardiac surgery procedures performed in the UK. Its crucial function is benchmarking surgical practice. From the NACSA database, we identified patients undergoing isolated, non-emergent CABG or isolated, non-emergent surgical aortic valve replacement (AVR) with available information regarding the time of surgery (am vs pm). The study period was from 2014 to 2018 . The operation was classed as am if the start time was in the 8 to 12 interval and pm if the start occurred 12:01 to 17:00. We have excluded emergency surgery. The primary outcome was in-hospital mortality. Missing or conflicting data for this outcome were obtained via record linkage to the Office for National Statistics census database.

Categorical variables were summarised as counts and percentages. Continuous variables were summarised as mean and standard 
deviation (SD) or median and interquartile range. Confounders considered included: age, gender, chronic obstructive pulmonary disease, peripheral vascular disease, diabetes, previous heart surgery, creatinine $>200 \mu \mathrm{mol} / \mathrm{l}$, left ventricular ejection fraction, previous myocardial infarction, pulmonary hypertension, deprivation index, days of the week. To investigate the effect of am/pm on the primary endpoint, we fitted a generalised linear mixed-effect model (GLMM) with hospitals included in the model as a random effect (random intercepts). The marginal R-squared considers only the variance of the fixed effects, while the conditional R-squared takes both the fixed and random effects into account. Effect estimates for fixed terms were reported as odds ratio (OR) and relative 95\% confidence interval. The association between significant random effects and the adjusted risk of the primary endpoint was reported as variance $(\sigma 2)$. P-value $<0.05$ was considered significant in all the analysis. All analyses were performed in $\mathrm{R}$ version 4.0.0. Ime4, sjPlot.

\subsection{RIPC post-hoc trial analysis}

The aim of the RIPC trial was to assess the effect of remote ischaemic preconditioning (RIPC) in 124 patients undergoing isolated CABG and AVR on cardiac injury, metabolic stress and inflammatory response [12]. The trial's primary endpoint was cardiac troponin I measured at baseline (before the operation) and at 6 h, 12 h, 24 h, 48 $\mathrm{h}$ and $72 \mathrm{~h}$ after the aortic cross-clamp release. Secondary endpoints were the analysis of adenine nucleotides onthe left and right ventricle myocardial biopsies (obtained using Trucut myocardial biopsies pre and post-reperfusion). Energy charge was calculated as follows: energy charge $=\mathrm{ATP}+(0.5 \mathrm{ADP}) / \mathrm{ATP}+\mathrm{ADP}+\mathrm{AMP}[13]$. In the original trial, there was no effect of the intervention (RIPC) compared to the sham. Therefore, we have included in the post-hoc analysis both arms of the study (sham and intervention) and performed the analysis of am and pm surgery. Morning surgery was considered any cross-clamp start time in the interval of 8-12:00 and afternoon if the start time was after 12:01. No patients in the study had out of hours surgery (e.g. cross-clamp start time after 17:00). For the analysis, we have used GraphPad Prism version 8.4.3 GraphPad Software, La Jolla California USA, www.graphpad.com. Because cardiac troponin time point data were missing per subject, we performed the analysis by fitting a mixed model. Similarly, we have fitted a mixed model for the myocardial nucleotide analysis. For the analysis of the baseline characteristics of the am and pm groups, we used $\mathrm{R}$ version 4.0.0, Table One package. For categorical variables analysis, we used for the chi-square test (with continuity correction) and for continuous variables a t-test. All the analysed data was anonymised; hence there was no need to obtain ethics approval in addition to original ethics approval for this post hoc analysis. The RIPC trial approved by the London-Harrow Research Ethics Committee (reference number REC number 12/LO/1361) and was registered to the International Standard Randomized Controlled Trial Number (ISRCTN) registry with the ID 33084113 (doi: 10.1186/ISRCTN33084113).

\section{Role of funding source}

Funding: None

\section{Results}

\subsection{Metaanalysis}

Our search identified a total of 965 references published between 2009 and 2020, of which five studies met included in this metaanalysis. One study presented two non-overlapping cohorts, one randomised group and one propensity-score-adjusted group [3]. The remaining four studies reported on PS-adjusted outcomes. Study characteristics are reported in Supplementary Table 2. Three studies were in Europe [3,6,7], one in Asia [8], and one in the USA [8]. The most performed procedure was CABG: one study reported on offpump CABG, two on isolated CABG or AVR $\pm C A B G$, one on CABG/AVR cohort, and one reported only patients undergoing AVR. Sample size ranged from 88 to 7791 patients. Operative characteristics in each study are presented in Supplementary Table 2. The assessment of the quality of the individual studies is reported in Supplementary Table 1. A summary of the risk of bias using the Newcastle- Ottawa Scale for the propensity-matched studies is found in Supplementary material (Supplementary Table 1). Three studies had a "very good" quality, and two were rated as "good" quality. The risk of the bias of the only RCT we have included was judged as low (Montaigne et al. 2018).

In the metaanalysis, we have only included the event number post propensity score matching or randomisation. Weighted pooled estimates for mortality and MI are summarised in Figs. 1 and 2, respectively. There was no difference in MI between patients operated in pm compared to am (OR 1.00, 95\% CI:0.64-1.57, $\mathrm{I}^{2}-62 \%$ ). There was moderate heterogeneity across studies. Similarly, no difference in mortality between pm vs am surgery was found (OR 1.10, 95\% CI:0.85-1.42, $\left.\mathrm{I}^{2}-0 \%\right)$. Funnel plots summarising the risk of bias, along with Egger's tests can be found in (Supplementary Material Figure 2 and 3).

\subsection{NACSA audit dataset analysis}

A total of 105459 patients were identified within the NACSA database. Of those, 78232 underwent non-emergent, isolated CABG and 27227 non-emergent, isolated SAVR. Unadjusted, baseline characteristics of these two cohorts operated in morning or afternoon are presented in Table 1 and 2. In the AVR cohort, patients operated on during pm hours $(11490,42 \%)$ resulted in being older but less likely to have poor left ventricular ejection fraction, MI, extracardiac arteriopathy, renal dysfunction and pulmonary hypertension. CABG patients operated on during am hours (45999, 59\%) showed a higher risk profile, being more likely to present with critical perioperative state and other comorbidities, such as recent MI, extracardiac arteriopathy and neurological dysfunction. There was no difference in the incidence of in-hospital mortality in the CABG cohort between patients operated during am vs pm hours (1.2\% vs $1.0 \%)$, whereas patients undergoing SAVR during am hours were more likely to experience this outcome (1.7\% vs $1.3 \%)$. Patient-level analysis of the NACSA dataset showed no effect of pm surgery on mortality in both the isolated AVR cohort (OR 0.82, 95\% CI: $0.65-1.03, \mathrm{p}=0.094)$ and the isolated CABG cohort (OR 0.94,95\% Cl:0.81 - 1.09, p=0.425) (Table 3).

\subsection{RIPC post-hoc trial analysis}

Baseline characteristics and operative data of patients in the RIPC trial stratified by daytime of the procedure are reported in Supplementary Table 4 . No significant difference between the two groups was found.

Our analysis did not show any effect of time surgery on the cardiac troponin release at the various time points post-reperfusion in the AVR group ( $\mathrm{p}=0.29$ ) or CABG group ( $\mathrm{p}=0.96$ ) (Fig. 3). Furthermore, there was no significant effect of time of surgery on ATP/AMP ratio $(\mathrm{p}=0.7484)$ (Fig. 4A), ATP/ADP ratio $(\mathrm{P}=0.7662)$ (Fig. 4B), and cardiomyocytes energy charge ( $p=0.3165$ ) (Fig. $4 C$ ) in the left and right ventricular myocardial biopsies pre- and post-reperfusion. An analysis by procedure type also showed no significant changes in nucleotide metabolism in AVR or CABG groups (Supplementary material Figure 4). 


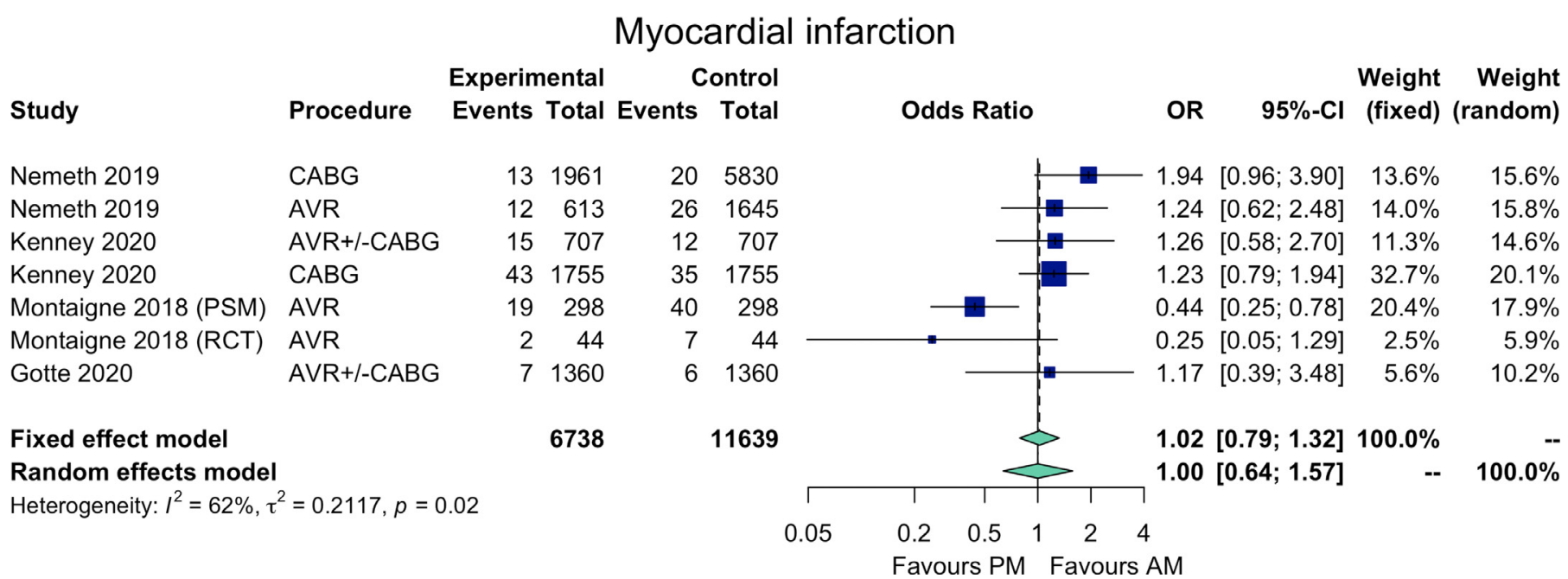

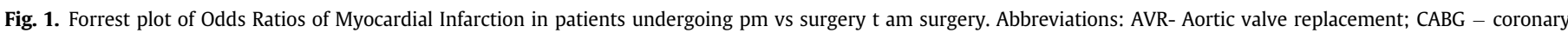
artery by-pass grafting; PSM - propensity matched study; RCT - srandomised controlled trial.

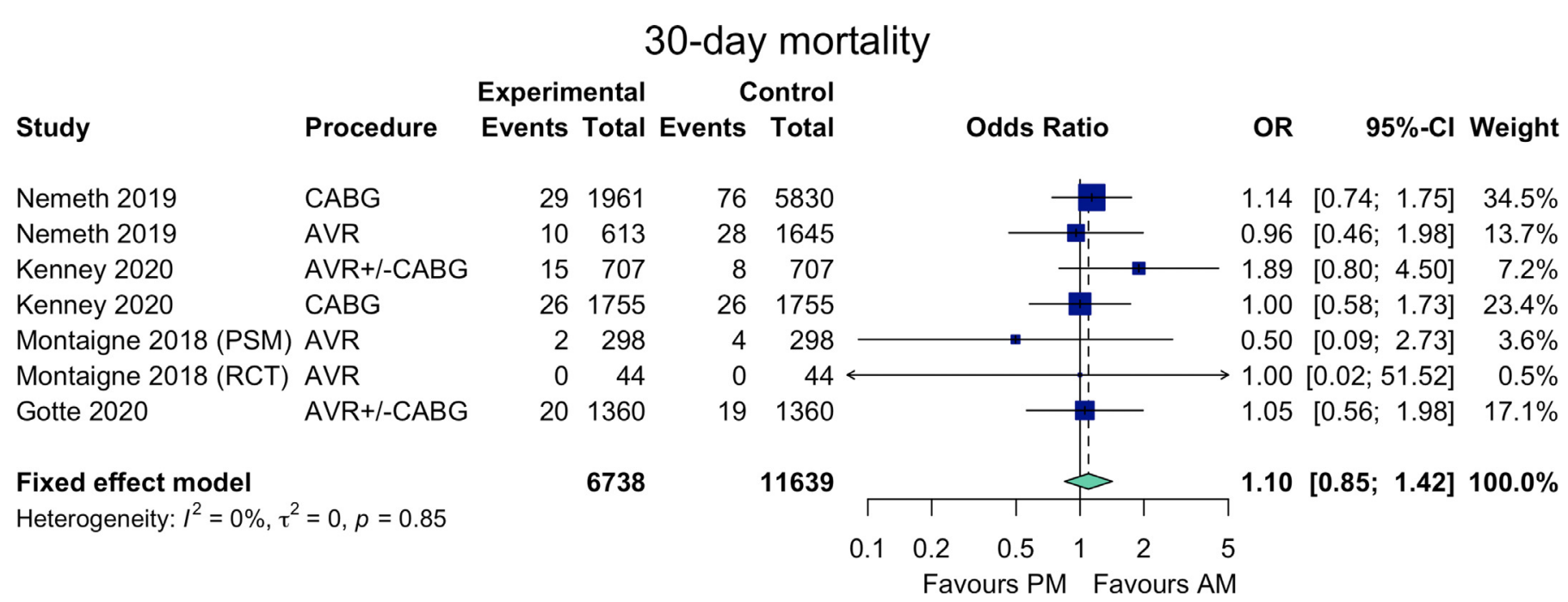

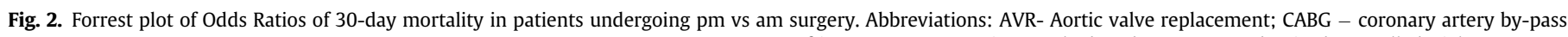
grafting; PSM - propensity matched study; RCT - srandomised controlled trial.

Table 1

Isolated AVR cohort baseline characteristics

\begin{tabular}{llll}
\hline Characteristic & AM & PM & P value \\
\hline Number of patients & 15737 & 11490 & \\
Age imputed & $69.14(11.96)$ & $70.06(11.14)$ & $<0.001$ \\
Female & $6590(41.9)$ & $4891(42.6)$ & 0.259 \\
Chronic pulmonary disease & $1582(10.1)$ & $1143(9.9)$ & 0.791 \\
Extracardiac arteriopathy & $976(6.2)$ & $609(5.3)$ & 0.002 \\
Diabetes & $597(3.8)$ & $402(3.5)$ & 0.213 \\
Previous cardiac surgery & $1268(8.1)$ & $388(3.4)$ & $<0.001$ \\
Creatinine $>200 \mu$ mol/l & $229(1.5)$ & $119(1.0)$ & 0.003 \\
Moderate LV & $2638(16.8)$ & $1690(14.7)$ & $<0.001$ \\
Poor LV & $698(4.4)$ & $421(3.7)$ & 0.002 \\
Recent MI & $263(1.7)$ & $154(1.3)$ & 0.032 \\
Pulmonary hypertension & $2638(16.8)$ & $1690(14.7)$ & $<0.001$ \\
Index of Multiple Deprivation Decile & $698(4.4)$ & $421(3.7)$ & 0.002 \\
Death & $264(1.7)$ & $146(1.3)$ & 0.008 \\
\hline
\end{tabular}

\section{Discussion}

To achieve homeostasis despite the recurring challenges in the environment, the human body displays a cyclic variation in its physiology or behaviour. Originating from the Latin' circa diem' or about a
Table 2

Isolated CABG cohort baseline characteristics

\begin{tabular}{llll}
\hline Characteristic & AM & PM & P value \\
\hline Number of patients & 45999 & 32233 & \\
Age imputed & $66.26(10.33)$ & $66.27(10.14)$ & 0.905 \\
Female & $8684(18.9)$ & $5889(18.3)$ & 0.032 \\
Chronic pulmonary disease & $3838(8.3)$ & $2712(8.4)$ & 0.737 \\
Extracardiac arteriopathy & $5247(11.4)$ & $3673(11.4)$ & 0.969 \\
Diabetes & $1505(3.3)$ & $952(3.0)$ & 0.013 \\
Previous cardiac surgery & $612(1.3)$ & $343(1.1)$ & 0.001 \\
Creatinine $>>200 \mu$ mol/l & $735(1.6)$ & $524(1.6)$ & 0.783 \\
Moderate LV & $10570(23.0)$ & $7112(22.1)$ & 0.003 \\
Poor LV & $1859(4.0)$ & $1227(3.8)$ & 0.101 \\
Recent MI & $15462(33.6)$ & $9916(30.8)$ & $<0.001$ \\
Pulmonary hypertension & $6943(15.1)$ & $5293(16.4)$ & $<0.001$ \\
Index of Multiple Deprivation Decile & $5.56(2.85)$ & $5.48(2.84)$ & $<0.001$ \\
Deaths & $534(1.2)$ & $335(1.0)$ & 0.117 \\
\hline
\end{tabular}

day, circadian rhythmicity is found not only in animals but also in plants, fungi and bacteria. This circadian rhythm is endogenous and encoded at a molecular level, including the cardiomyocyte. One hypothesis is that circadian clock genes can independently influence outcomes after cardiac surgery by affecting the ischaemic tolerance 
Table 3

Results of generalised linear mixed model (binomial) for the primary endpoint (mortality) among patients undergoing isolated AVR and CABG.

\begin{tabular}{|c|c|c|c|c|c|c|}
\hline \multirow[b]{2}{*}{ Predictors } & \multicolumn{3}{|c|}{ AVR } & \multicolumn{3}{|c|}{ CABG } \\
\hline & Odds Ratios & $\mathrm{CI}$ & $\mathrm{P}$ & Odds Ratios & $\mathrm{CI}$ & $\mathrm{p}$ \\
\hline Intercept & 0.00 & $0.00-0.00$ & $<0.001$ & 0.00 & $0.00-0.00$ & $<0.001$ \\
\hline Age imputed & 1.04 & $1.03-1.05$ & $<0.001$ & 1.06 & $1.05-1.07$ & $<0.001$ \\
\hline Female & 1.54 & $1.24-1.93$ & $<0.001$ & 1.60 & $1.35-1.89$ & $<0.001$ \\
\hline Chronic pulmonary disease & 2.08 & $1.57-2.76$ & $<0.001$ & 1.62 & $1.32-1.99$ & $<0.001$ \\
\hline Extracardiac arteriopathy & 1.44 & $1.00-2.08$ & 0.052 & 1.82 & $1.52-2.17$ & $<0.001$ \\
\hline NDD & 1.28 & $0.78-2.12$ & 0.328 & 1.52 & $1.11-2.07$ & 0.009 \\
\hline Previous cardiac surgery & 3.14 & $2.31-4.26$ & $<\mathbf{0 . 0 0 1}$ & 4.01 & $2.81-5.71$ & $<0.001$ \\
\hline Creatinine $>200 \mu \mathrm{mol} / \mathrm{l}$ & 4.23 & $2.52-7.10$ & $<0.001$ & 4.12 & $3.09-5.50$ & $<0.001$ \\
\hline Moderate LV & 1.58 & $1.21-2.05$ & 0.001 & 2.17 & $1.67-2.84$ & $<0.001$ \\
\hline Poor LV & 1.81 & $1.16-2.83$ & 0.009 & 1.70 & $1.31-2.20$ & $<0.001$ \\
\hline Recent MI & 1.01 & $0.47-2.18$ & 0.979 & 1.68 & $1.42-1.98$ & $<0.001$ \\
\hline Pulmonary hypertension & 2.01 & $1.57-2.57$ & $<0.001$ & 1.17 & $0.93-1.47$ & 0.177 \\
\hline Index of Multiple Deprivation Decile & 0.97 & $0.94-1.01$ & 0.183 & 0.97 & $0.94-0.99$ & 0.013 \\
\hline Surgery in the pm & 0.82 & $0.65-1.03$ & 0.094 & 0.94 & $0.81-1.09$ & 0.425 \\
\hline \multicolumn{7}{|l|}{ Random Effects } \\
\hline \multicolumn{7}{|l|}{$\sigma 2$} \\
\hline & 3.29 & & & 3.29 & & \\
\hline$\tau 00$ hospital & 0.07 & & & 0.07 & & \\
\hline ICC & 0.02 & & & 0.02 & & \\
\hline N hospital & 32 & & & 34 & & \\
\hline Observations & 22880 & & & 68368 & & \\
\hline Marginal R2 / Conditional R2 & $0.145 / 0.164$ & & & $0.200 / 0.216$ & & \\
\hline
\end{tabular}

Aortic valve replacement
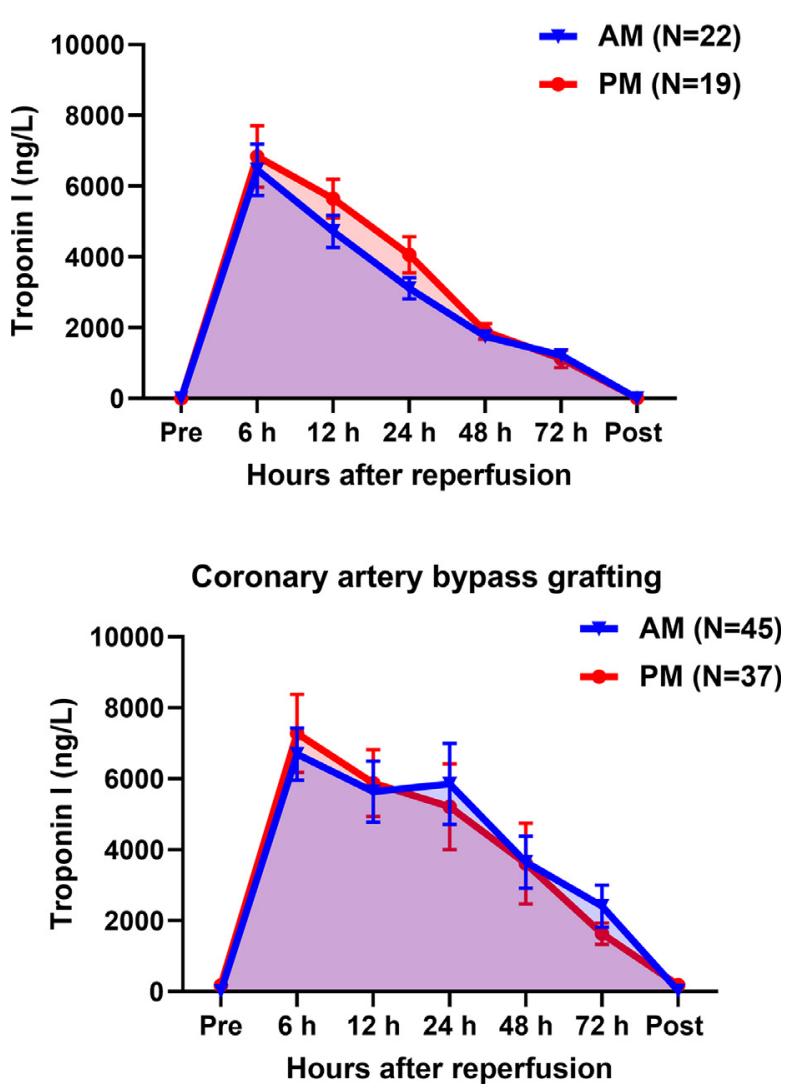

Fig. 3. Cardiac Troponin I release in the am vs pm surgery cohorts at the seven-time points (hours post-reperfusion). Data are summarised as mean \pm SEM.

of the myocardium in relation to the time of day. Therefore, our first aim was to synthesise the available evidence on this topic. In our metaanalysis of propensity studies and a randomised study, we demonstrate no effect of time of surgery on the relevant hardcore endpoints of myocardial infarction and operative mortality. Montaigne et al. [3] is the only study that showed some benefit of pm surgery in terms of perioperative myocardial infarction rates based on propensity-matched cohorts of AVR patients. However, this study is in contrast with the rest of the studies published afterwards, that show no effect of daytime of surgery. Our metaanalysis is mainly based on propensity-matched studies. We found only one small randomised controlled trial, part of Montaigne et al. study [3] that had no events for mortality and no significant difference in perioperative myocardial infarction rates.

Access to the NICOR UK adult cardiac database allowed us to perform a retrospective adjusted analysis to assess the effect of am vs pm surgery on mortality in a large cohort, reflecting real-world practice. Similar to our metaanalysis results, we found no effect daytime of surgery in either CABG or AVR cohorts. Compared to previous studies, our risk adjustment model considers the social, economic status (Index of Multiple Deprivation Decile). This measure is known to affect outcomes after cardiac surgery [14]. Furthermore, circadian rhythmicity can be affected by social and economic status [15]; therefore, a relevant parameter to adjust for. Finally, in the small randomised trial by Montaigne et al. [3], there was a significantly lower myocardial injury, as measured by cardiac troponin, in patients undergoing aortic valve replacement surgery in the afternoon. This finding was not confirmed in the subsequent studies that were all non-randomised. Götte et al. [6] found a contradicting result in a propensity-matched analysis where there was a more pronounced release of troponin in the morning AVR group compared to the afternoon group. In the study by Baik et al. [5], there was no significant daytime troponin variation in patients undergoing off-pump CABG. We addressed this hypothesis in a posthoc trial database analyses, where we found no difference in troponin release in patients undergoing am or pm surgery. This was subsequently confirmed in the analysis of adenine nucleotide from ventricular biopsies.

The current work has several limitations. Firstly, we conducted our metaanalysis mainly in non-randomised studies. Only five studies met the inclusion, which could affect the power of the metaanalysis and assessment of publication bias. While almost all studies were adjusted by propensity matching, such type of metaanalysis is limited by the increased clinical heterogeneity and methodological heterogeneity among the studies that are included [16]. Some studies used various definitions for the MI and mortality endpoints (Supplementary Table 4). Furthermore, the definition of am or pm case 
A

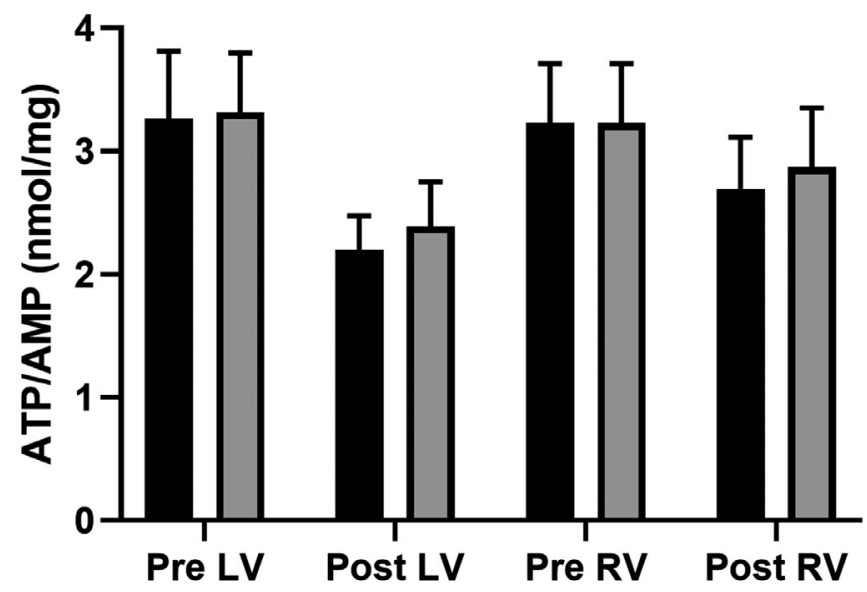

B
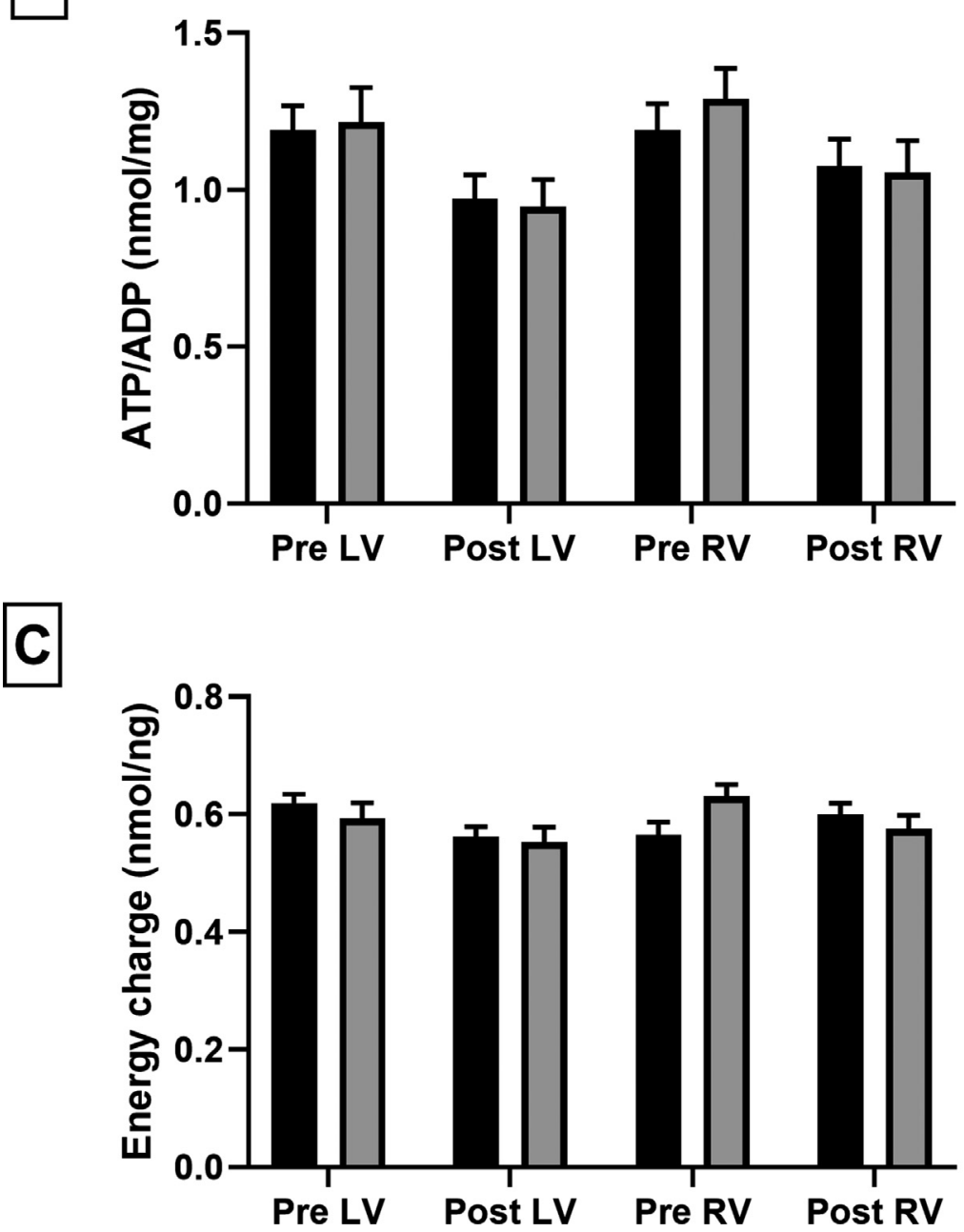

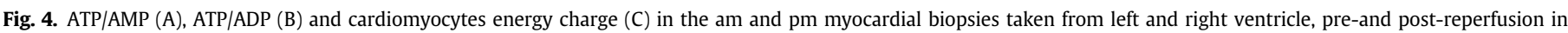

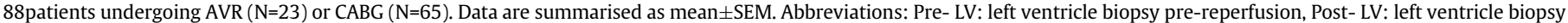
post-reperfusion; Pre-RV: right ventricle biopsy pre-reperfusion; Post- RV: right ventricle biopsy post-reperfusion

differed (Supplementary Table 5). The analysis of the NACSA audit dataset is limited by the retrospective analysis of prospectively collected data regardless of robust adjustment we implemented. Specific limitations to this analysis are errors in data entry that are inherent to administrative databases. We also have to acknowledge that original animal experiments assessed myocardial tolerance over 24 hours. The available clinical studies, including our work, compared the morning and afternoon surgery outcomes within an approximately 12 -hour time frame. Looking retrospectively at operations performed over a 24-hour time frame introduces selection 
bias because we risk comparing surgeries performed in the morning with higher risk cases performed out of hours. Another perspective is to look at myocardial ischaemic tolerance with a higher resolution, whereby surgeries could be compared according to hourly time frame starting times over a more extended period. Perhaps differences are observed for surgeries starting between 9-10 pm versus 8-10 am for example.

It was not possible to adjust the analysis according to the surgeon experience or various techniques used in the surgical centres that could affect outcomes. For example, one study [3] had stringent criteria to control for surgeon experience and analysed cases performed in the am versus pm by the same team on the same list (Supplementary Table 5 ). We have performed our analysis on a trial quality dataset where there was no effect of the intervention. However, posthoc analyses have several limitations [17]. In our case, the randomisation of the trial population was performed according to another type of intervention (e.g., not the daytime of surgery). Finally, our study lacks follow-up of data on the myocardial function in patients that had surgery in the am versus the pm group. It is noteworthy that the original study by Montaigne followed heart failure incidence over 500 days post-surgery and found a significant divergence between the am and pm groups [3]

\section{Conclusion}

In this three-part analysis, we found no evidence that the time of procedure morning or afternoon influences mortality or myocardial injury in patients undergoing cardiac surgery.

\section{Contributors}

Daniel P Fudulu: conceptualisation, data curation, formal analysis, investigation, methodology, project administration, resources, software, supervision, validation, visualisation, writing - original draft, and writing - review and editing.

Arnaldo Dimagli: data curation, formal analysis, methodology, project administration, writing - original draft, and writing - review and editing.

Lauren Dixon: data curation, methodology, writing - review and editing.

Manraj Sandhu: data curation, methodology, writing - review and editing.

Lucia Cocomello: data curation, methodology, writing - review and editing.

Gianni D Angelini: conceptualisation, methodology, project administration, resources, software, supervision, validation, visualisation, writing - original draft, and writing - review \& editing

Umberto Benedetto: conceptualisation, data curation, formal analysis, investigation, methodology, project administration, resources, software, supervision, validation, visualisation, writing - original draft, and writing - review and editing.

\section{Data sharing statement}

Requests for data should be directed to the lead author (daniel. fudulu@bristol.ac.uk). Requests will be assessed for scientific rigour before being granted. Data will be anonymised and securely transferred. A data sharing agreement will be required.

\section{Declaration of competing interest}

No conflicts of interest to disclose.

\section{Acknowledgements}

This study was supported by the NIHR Biomedical Research Centre at University Hospitals Bristol and Weston NHS Foundation Trust, and the University of Bristol. The views expressed are those of the author(s) and not necessarily those of the NIHR or the Department of Health and Social Care.

\section{Supplementary materials}

Supplementary material associated with this article can be found, in the online version, at doi:10.1016/j.lanepe.2021.100140.

\section{References}

[1] Muller JE, Stone PH, Turi ZG, Rutherford JD, Czeisler CA, Parker C, et al. Circadian variation in the frequency of onset of acute myocardial infarction. $\mathrm{N}$ Engl J Med [Internet] 1985 Nov 21;313(21):1315-22 [cited 2020 Oct 7]Available from: https://pubmed.ncbi.nlm.nih.gov/2865677/.

[2] Durgan DJ, Pulinilkunnil T, Villegas-Montoya C, Garvey ME, Frangogiannis NG, Michael LH, et al. Short communication: ischemia/reperfusion tolerance is timeof-day-dependent: mediation by the cardiomyocyte circadian clock. Circ Res [Internet] 2010 Feb 19;106(3):546-50 2009/12/10Available from: https:// pubmed.ncbi.nlm.nih.gov/20007913.

[3] Montaigne D, Marechal X, Modine T, Coisne A, Mouton S, Fayad G, et al. Daytime variation of perioperative myocardial injury in cardiac surgery and its prevention by Rev-Erb $\alpha$ antagonism: a single-centre propensity-matched cohort study and a randomised study. Lancet 2018.

[4] Sokullu O, Sanioğlu S, Kurc E, Sargin M, Deniz H, Tartan Z, et al. Does the circadian rhythm of melatonin affect ischemia-reperfusion injury after coronary artery bypass grafting? Heart Surg Forum 2009.

[5] Baik J, Nam J, Oh J, Kim GW, Lee E, Lee Y, et al. Effect of operative time on the outcome of patients undergoing off-pump coronary artery bypass surgery. J Card Surg [Internet] 2019 Nov 26;34(11):1220-7 [cited 2020 Oct 7]Available from: https://onlinelibrary.wiley.com/doi/abs/10.1111/jocs.14231.

[6] Götte J, Zittermann A, Deutsch MA, Schramm R, Bleiziffer S, Hata M, et al. Daytime variation in aortic valve surgery and clinical outcome: a propensity score- matched analysis. Ann Thorac Surg 2020.

[7] Kenney PS, Nielsen PH, Modrau IS. Daytime-Dependent Cardioprotection in cardiac surgery: a large propensity-matched cohort study. Ann Thorac Surg 2020.

[8] Nemeth S, Schnell S, Argenziano M, Ning Y, Kurlansky P. Daytime variation does not impact outcome of cardiac surgery: results from a diverse, multi-institutional cardiac surgery network. J Thorac Cardiovasc Surg 2020.

[9] Moscarelli M, Fiorentino F, Suleiman MS, Emanueli C, Reeves BC, Punjabi PP, et al. Remote ischaemic preconditioning in isolated aortic valve and coronary artery bypass surgery: a randomized trial. Eur J Cardio-thoracic Surg 2019;55(5): 905-12.

[10] DerSimonian R, Laird N. Meta-analysis in clinical trials. Control Clin Trials 1986.

[11] Cox DR. The continuity correction. Biometrika 1970.

[12] Moscarelli M, Fiorentino F, Suleiman M-S, Emanueli C, Reeves BC, Punjabi PP, et al. Remote ischaemic preconditioning in isolated aortic valve and coronary artery bypass surgery: a randomized trialdagger. Eur J Cardiothorac Surg 2019 May;55 (5):905-12.

[13] Abdul-Ghani S, Fleishman AN, Khaliulin I, Meloni M, Angelini GD, Suleiman MS Remote ischemic preconditioning triggers changes in autonomic nervous system activity: implications for cardioprotection. Physiol Rep [Internet], 5; 2017 Feb 1. [cited 2021 Jan 12]Available from: /pmc/articles/PMC5309573/?report=abstract.

[14] Benedetto U, Dimagli A, Gibbison B, Sinha S, Pufulete M, Fudulu D, et al. Disparity in clinical outcomes after cardiac surgery between private and public (NHS) payers in England. Lancet Reg Heal - Eur [Internet]; 000. Available from:. doi: 10.1016/j.lanepe.2020.100003.

[15] Walker WH, Walton JC, DeVries AC, Nelson RJ. Circadian rhythm disruption and mental health. Translational Psychiatry 2020.

[16] Metelli S, Chaimani A. Challenges in meta-analyses with observational studies. Evid Based Ment Health [Internet], 23; 2020 May 1. [cited 2021 Jan 18]Available from:p. 83-7http://ebmh.bmj.com/.

[17] Curran-Everett D, Milgrom H. Post-hoc data analysis: benefits and limitations. Curr Opin Allergy Clin Immunol 2013;13(3):223-4. 